\title{
Genotype-phenotype correlation in inherited brain myelination defects due to proteolipid protein gene mutations
}

\author{
Fabrice Cailloux, Fernande Gauthier-Barichard, Corinne Mimault, Valerie Isabelle, \\ Virginie Courtois, Geneviève Giraud, Bernard Dastugue, Odile Boespflug-Tanguy and \\ Clinical European Network on Brain Dysmyelinating Disease ${ }^{\dagger}$
}

INSERM U384, Faculté de Médecine, Clermont-Ferrand, France

Pelizaeus-Merzbacher disease (PMD) and spastic paraplegia type 2 (SPG2) are X-linked developmental defects of myelin formation affecting the central nervous system (CNS). They differ clinically in the onset and severity of the motor disability but both are allelic to the proteolipid protein gene (PLP), which encodes the principal protein components of CNS myelin, PLP and its spliced isoform, DM20. We investigated 52 PMD and 28 SPG families without large PLP duplications or deletions by genomic PCR amplification and sequencing of the PLP gene. We identified 29 and 4 abnormalities respectively. Patients with PLP mutations presented a large range of disease severity, with a continuum between severe forms of PMD, without motor development, to pure forms of SPG. Clinical severity was found to be correlated with the nature of the mutation, suggesting a distinct strategy for detection of PLP point mutations between severe PMD, mild PMD and SPG. Single amino-acid changes in highly conserved regions of the DM20 protein caused the most severe forms of PMD. Substitutions of less conserved amino acids, truncations, absence of the protein and PLP-specific mutations caused the milder forms of PMD and SPG. Therefore, the interactions and stability of the mutated proteins has a major effect on the severity of PLP-related diseases. European Journal of Human Genetics (2000) 8, 837-845.

Keywords: proteolipid protein gene (PLP); Pelizaeus Merzbacher disease (PMD); spastic paraplegia (SPG); myelin disorder; $\mathrm{X}$ chromosome; molecular diagnosis

\section{Introduction}

The distinction between inherited defects in central nervous system (CNS) myelination (dysmyelinating diseases) and genetic diseases leading to myelin destruction (demyelinat-

Correspondence: Odile Boespflug-Tanguy, INSERM U384, Faculté de Médecine, 28, place Henri Dunant, BP 38, 63001 Clermont-Ferrand, Cedex, France. Tel: + 33473608024 ; Fax: + 33473276132 ; E-mail: odile.boespflug@inserm.u-clermont1.fr

${ }^{\dagger}$ ENBDD: Clinical European Network on Brain Dysmyelinating Disease: M Baethmann, E Bertini, B Chabrol, JM Cuisset, J Gaertner, F Hanefeld, A Kohlschutter, P Landrieu, JM Lopes-Terradas, M Mayer, S Peudenier, JM Prats-Vinas, D Rodriguez, D Rating, R Surtees, G Uziel, L Vallee and T Voit, with the collaboration of J Aicardi, P Amarenco, I Bernard, J Boulloche, ML Chauvet, G Cioni, I Desguerre, C DeSouza, D Fontan, A Gal, C Hübner, I Krägeloh-Mann, G Kurlemann, D Lacombe, C LeBerre, B Lemarec, J Lopez-Pison, C Moraine, K Muller, A Nivelon-Chevalier, JP Nuyts, K Pohl, E Scalais, W Schrank, G Sebire, M Spada, H Steinbock, S Stöcker, M Tardieu, A Toutain and L Zelante

Received 23 July 1999; revised 5 June 2000; accepted 20 June 2000 ing diseases) was first made at the beginning of the twentieth century by Pelizaeus and Merzbacher, who described a dysmyelinating disease that became the prototype of this class of disorders. They defined this disease as involving (i) early-onset motor disability inherited via an X-linked recessive trait and (ii) a neuropathological pattern of myelin sheath disappearance strictly limited to the CNS, with no nerve cell or axon involvement and no inflammation.

Introduction of electrophysiological and magnetic resonance imaging (MRI) techniques to demonstrate the abnormal formation of CNS myelin, defined new diagnostic criteria for PMD. ${ }^{1}$ The proteolipid protein gene (PLP) was identified as the causative locus for PMD. ${ }^{2}$ This gene, which maps to Xq22, encodes two proteolipid proteins in oligodendrocytes, PLP and its spliced isoform, DM20, which account for more than $50 \%$ of the total protein mass of CNS myelin. 
PLP is a major structural component of CNSmyelin, ${ }^{3}$ whereas DM 20 which is produced earlier in CNS development may be involved in oligodendrocyte differentiation and survival. ${ }^{4,5} \mathrm{~A}$ wide spectrum of PLP mutations have been found in animal model $s^{6}$ and PMD patients: duplication of the entire PLP gene is the most frequent causative mutation, ${ }^{7,8}$ with other mutations in the coding and non-coding regions of PLP accounting for 10 to $25 \%$ of cases. ${ }^{9}$ PLP gene mutations have also been associated with $\mathrm{X}$-linked spastic paraplegia2 (SPG2). ${ }^{10-12}$ This disease involves early-onset progressive gait difficulties associated in most patients with neurological signs classically found in PMD patients (nystagmus, ataxia, mild mental retardation), defining complicated forms of SPG2.

However, SPG2 differs from PMD in that motor milestone achievement is almost normal in the first year of life for SPG2. We investigated 52PMD and 28SPG families with no large PLP duplications or del etions by PCR amplification and sequencing of the seven coding regions and the splice sites of PLP. Abnormalities were identified in 29PMD and 4SPG2 families, missense mutations being the most common.

The 52 PLP-mutated patients presented a large range of disease severity, which we graded according to the best motor function achieved between 1 and 10 years of age. Clinical severity was found to be correlated with the nature of the mutation, suggesting a distinct strategy for detection of PLP point mutations between severe PMD, mild PMD and SPG. The interactions and stability of the mutated proteins was found to have a major effect on the severity of PLP-related diseases.

\section{Patients \\ Selection of patients}

Patients with PMD or SPG were referred to us by paediatric neurologists from various European countries.

104 PMD patients were selected for PLP analysis based on clinical, electrophysiological and magnetic resonance imaging criteria described elsewhere. ${ }^{2}$ The 52 patients with no large PLP duplications or deletions were eligible for PLP analysis by PCR amplification and sequencing of the coding and splicing regions of the gene.

Twenty-nine SPG patients were selected according to the following clinical criteria:

(i) male,

(ii) early-onset ${ }^{5}$ PG (before 10 years of age), with almost normal milestone achievement during the first year of life,

(iii) slow progression,

(iv) low CNS conduction velocities on somatosensory or brainstem evoked potential evaluation, and/or 'hypomyelinated' areas of the brain on MRI (iso or hyper T1 associated with hyper T2 signals of the white matter relative to the grey matter),

(v) no other known cause (including normal results for: spinal cord MRI, amino acid and organic acid gas chromatography, lactate/pyruvate ratio, Iysosomal enzymes, very long chain fatty acids, HTLV1 antibodies).

The 28 patients with no large PLP duplications or deletions were eligible for subsequent PLP analysis.

\section{Classification of PLP-mutated families}

In the 33 families with identified PLP mutations, detailed clinical information was collected from 52 of the 56 patients reported to be affected. These patients had a mean age of $18.5+/-17.5$ years at the time of clinical evaluation.

The PMD group ( 42 patients) presented early impairment (before 3 months) of normal motor development associated with neurological signs, which were gradually modified by the maturing nervous system: early nystagmus was observed in all patients, except family 35 , at a mean age of $1.2+/-1.5$ months; bobbing movements of the head and trunk, choreoathetotic movements of the limbs and cerebellar ataxia were observed between 6 and 18 months of age; progressive spastic paraplegia with pyramidal tract signs were clearly present after 2-4 years. In all cases, motor handicap was greater than impai rment of psycho-intel lectual development. No rapid degradation was observed. Few patients had no motor acquisition but the performance of most improved slowly until age 10-12 years. Performance differed between patients. After reaching a plateau, a slow deterioration was observed after 10-20 years, accompanied by the development of cortical atrophy on MRI. Five patients died (12\%) at a mean age of $30+/-11$ years (range: 3-60 years).

We classified patients into five forms according to the best motor function they were able to achieve between the ages of 1 and 10 years:

- form 0 included 8 patients (19\%), with a mean age of $5.6+/-2.3$ years at clinical evaluation, with no motor achievement; one presented stridor (family 49), one (family 26) had seizures well controlled by anti-epileptic drugs and one died at age 3 (family 107);

- form 1 included 12 patients (28\%), with a mean age of $8.3+/-5$ years at clinical evaluation, who achieved head control between 2 and 4 years; one died at 10 years (family 29);

- form 2 included 6 patients (14\%), with a mean age of $22.1+/-20$ at clinical evaluation, able to achieve a sitting position between 2 and 5 years; half achieved a dysarthric but comprehensible speech;

- form 3 included 13 patients (31\%), with a mean age of $19.3+/-15$ at clinical evaluation, able to achieve a sitting position between 1 and 2 years, to walk with 
support at a mean age of $3.5+/-1.5$ years (range $2-6$ ) but six lost this capacity between 14 and 20 years; all achieved dysarthric but comprehensible speech and seven were integrated into a normal school, using a typewriter to communicate; three died at a mean age of $45+$-/ 14 (range 25-60).

- form 4, autonomous walking. None of our PMD patients fell into this category.

MRI analysis in 33 cases, and electrophysiological (BAEP, SEP) analysis in 28 identified no significant differences between patients of the various groups.

For the 10SPG families analysed, spasticity started between 12 and 48 months (mean age $28.8+/-9.8$ months). Only two patients expressed a pure form of SPG (families 9 and 50). Associated neurological signs were early nystagmus (3), ataxia (7), and mild mental retardation (5). All, except one in family 50, achieved the motor milestones of the first year of life normally or slightly late, and started to walk at a mean age of $22+/-12$ months, 6 autonomously between 14 and 36 months and 4 with assistance between 24 and 48 months. Seven lost the capacity to walk at a mean age of $17+/-5$ years (range 7-28years). Two died, at the ages of 37 and 24 years.

For 10 families, clinical severity was evaluated for at least two affected patients. No major difference in disease severity was observed between affected individuals in the same family (Table1). However, in the two families with more than four patients each, we observed minor differences: in PMD family 1 , four patients were able to walk with support, whereas two only achieved the sitting position, and in SPG family 9, two patients were unable to walk without support, whereas the other three walked unaided. We used this intrafamilial homogeneity for disease severity in both PMD and SPG to determine whether there was a correlation between the disease severity and the type of PLP mutation. For each family, the severity of the disease was scored according to the clinical severity of the affected patient for sporadic forms and the clinical score of most of the affected sibs for familial forms. If scores were equal, the best motor function the patients were able to achieve was used. Forms 0 and 1 were considered to correspond to severe forms of PMD whereas forms 2 and 3 were considered to correspond to mild forms.

\section{Methods \\ PLP analysis}

Point mutations in PLP were investigated by sequence analysis. DNA was amplified using intronic primers flanking each exon of the human PLP gene, in standard conditions adapted for each set of primers, as previously described. ${ }^{13}$ Sequences were determined twice, with two independent PCR amplifications of the DNA from each individual.

\section{Amino-acid sequence comparison for DM proteins}

We compared the human DM20 sequence with sequences of other homologous DM proteins, including the DM20 of trout $^{14}$ and chicken ${ }^{15}$ the M6a, M6b proteins of humans ${ }^{16}$ and mice $^{17}$ and the $\mathrm{DM} \alpha, \mathrm{DM} \beta$ and $\mathrm{DM} \gamma$ proteins of Squalus. ${ }^{18}$ Amino acids 90 to $100 \%$ identical were considered to be highly conserved, those $60-89 \%$ identical, partially conserved and those less than $60 \%$ identical, not conserved.

\section{Results}

\section{Type and position of PLP mutations in PMD and SPG} patients

In the 52 PMD and 28 SPG families selected for sequencing of the seven coding regions and the exon/intron junctions of the PLP gene, we identified 33 abnormalities (29 in PMD patients, 4 in SPG patients) (Table1).

Twenty-three were missense mutations, three were deletion/insertions with frameshifts and seven were splice-site mutations. Mutations were in coding regions in $80 \%$ of PMD patients (24 of 29 cases): in exons2 (29\%), 4 (29\%) and 5 $(21 \%)$, whereas two of the mutations in SPG patients were in the PLP-specific coding region, exon $3 \mathrm{~B}$. No mutations were observed in exons1 and 7 of PLP.

Of the 23 aa changes resulting from missense mutations, $48 \%$ affected the C-D loop, whereas each of the other locations accounted for only 4 to $13 \%$ of the mutations. In the 29PMD families with PLP mutations, disease severity score was 0 in seven cases, 1 in 10 cases, and 2 in three cases and 3 in nine cases, whereas all four SPG families scored 4.

\section{Correlation between disease severity and type of mutation}

The type of mutation was considered in relation to the severity of the disease (Figure 1). Missense mutations were observed in two thirds of cases for the severe forms (types 0 and 1 ) and in one third of cases for the milder forms (types2, 3 and 4), whereas all other types of abnormality were observed al most exclusively in the mildest forms (types 3 and $4 ; 80 \%)$.

\section{Correlation between disease severity and the position of exonic mutations}

Of the 26 mutations in coding regions (Figure 1), fifteen were responsible for severe PMD forms (57\%), nine for mild PMD forms (35\%) and two for SPG form 4 (8\%). Mutations causing severe forms of PMD mapped essentially to exons2 (40\%) and $4(33 \%)$, more rarely to exons $6(13 \%), 5(7 \%)$, and $3 \mathrm{~A}$ (7\%), and never to exons $1,3 \mathrm{~B}$ and 7 . Mutations causing mild forms of PMD mapped predominantly to exon 5 (44\%), more rarely to exons $4(22 \%), 6(22 \%)$ and $2(12 \%)$. SPG mutations mapped exclusively to exon 3B. 
Table 1 Proteolipid protein gene (PLP) mutations identified by sequencing of the seven coding regions and the splice sites, in 33 families affected by X-linked dysmyelinating diseases (PMD, Pelizaeus Merzbacher Disease and SPG, X-linked spastic paraplegia type 2)

\begin{tabular}{|c|c|c|c|c|c|c|c|c|}
\hline Family no. & $\begin{array}{l}\text { Age at clinical } \\
\text { evaluation } \\
\text { (in years) }\end{array}$ & Phenotype & $\begin{array}{l}\text { Clinical severity } \\
\text { for each } \\
\text { patient }\end{array}$ & $\begin{array}{l}\text { Severity score } \\
\text { for the } \\
\text { family }\end{array}$ & PLP exon & Base change & $\begin{array}{l}\text { Amino acid } \\
\text { substitution }\end{array}$ & $\begin{array}{l}\text { Position in } \\
\text { PLP } \\
\text { DM-20 }\end{array}$ \\
\hline \multicolumn{9}{|c|}{ PLP missense mutations } \\
\hline 12 & 8 & PMD & $? / 1$ & 1 & 2 & \multirow{2}{*}{$\begin{array}{l}\text { CTG-CCG } \\
\text { TTC-TTA }\end{array}$} & L30P & A \\
\hline 11 & 8-9 & PMD & $0 / 0$ & 0 & 2 & & F31L & A \\
\hline 99 & 5 & PMD & 0 & 0 & 2 & TGT-TAT & CD34Y & $A-B$ \\
\hline 176 & 3 & PMD & 1 & 1 & 2 & GCC-ACC & A38T & $A-B$ \\
\hline 22 & 15 & PMD & 1 & 1 & 2 & ACA-ATA & T42I & $A-B$ \\
\hline 29 & $10-9$ & PMD & $1 / 1$ & 1 & 2 & ТTC-TCC & F50S & $A-B$ \\
\hline 106 & 5 & PMD & 2 & 2 & 2 & TAT-TGT & Y59C & B \\
\hline 107 & 3 & PMD & 0 & 0 & 3 & GGA-CGA & G73R & B \\
\hline 160 & $23-6$ & SPG & $4 / 4$ & 4 & 3B & CAT-TAT & H129Y & $B-C$ \\
\hline 21 & 12 & PMD & 1 & 1 & 4 & TAC-TGC & Y174C & C-D \\
\hline 209 & 18 & PMD & 4 & 1 & 4 & TGG-TGT & W180C & C-D \\
\hline 41 & 6 & PMD & 3 & 3 & 4 & $A C C-A A C$ & T182N & C-D \\
\hline 36 & 8 & PMD & 0 & 0 & 4 & GAC-GAA & D202E & $C-D$ \\
\hline 37 & 8 & PMD & 0 & 0 & 4 & GAT-GGT & D202G & C-D \\
\hline 49 & 5 & PMD & 0 & 0 & 4 & GAT-AAT & D202N & C-D \\
\hline 52 & 10 & PMD & 2 & 2 & 5 & TGG-CGG & W211R & C-D \\
\hline 24 & 16 & PMD & 2 & 2 & 5 & ССТ-ТCT & P215S & $C-D$ \\
\hline 53 & 7 & PMD & 2 & 2 & 5 & CCT-GCT & P215A & C-D \\
\hline $1 *$ & $\begin{array}{l}24-24-26 \\
50-45-50\end{array}$ & PMD & $\begin{array}{l}3 / 3 / 3 / \\
2 / 2 / ?\end{array}$ & 3 & 5 & GTT-TTT & V218F & $C-D$ \\
\hline 23 & 8 & PMD & 1 & 1 & 5 & \multirow{4}{*}{\multicolumn{2}{|c|}{$\begin{array}{l}\text { TGC-TAC } \\
\text { CAA-CCA } \\
\text { GGG- } \\
\text { GCA-GAA }\end{array}$}} & C-D \\
\hline 133 & 6 & PMD & 3 & 3 & 6 & & & D \\
\hline 143 & 3 & PMD & 1 & 1 & 6 & & & D \\
\hline \multirow[t]{3}{*}{217} & \multirow[t]{3}{*}{2} & \multirow[t]{3}{*}{ PMD } & \multirow[t]{3}{*}{1} & \multirow[t]{3}{*}{1} & 6 & & & $\mathrm{D}$ \\
\hline & & & & & & & & $\begin{array}{l}\text { Affected } \\
\text { domains of }\end{array}$ \\
\hline & & & & & Mutation in & Effect & tation & PLP/DM20 \\
\hline 9 & $29-11-34$ & SPG & $\begin{array}{l}3 / 3 / 4 / \\
4 / 4\end{array}$ & 4 & exon 3B & del G & & fs $136-144$ \\
\hline 3* & $12-34$ & PMD & $3 / 3$ & 3 & exon 4 & del + & & fs 195-233 \\
\hline 182 & 2 & PMD & 3 & 3 & exon 6 & ins $T$ & & fs $240-257$ \\
\hline 35 & $43-42-27$ & PMD & $3 / 3 / 3$ & 3 & intron 2 & asp ( & & - \\
\hline 26 & 8 & PMD & 0 & 0 & intron 3 & asp ( & & - \\
\hline 5 & $18-30-43-20$ & SPG & $4 / 4 / 4 / 4$ & 4 & intron 3 & asp ( & & - \\
\hline 198 & 2 & PMD & 3 & 3 & intron 5 & asp (c & & - \\
\hline 149 & $27-27$ & SPG & $4 / 4$ & 4 & intron 5 & del 2 & & - \\
\hline 62 & $8-4$ & PMD & $1 / 1$ & 1 & intron 6 & dsp ( & & - \\
\hline 221 & 3 & PMD & 3 & 3 & intron 6 & dsp ( & & - \\
\hline
\end{tabular}

Disease severity was scored for each patient according to the best motor function achieved between the ages of 1 and 10 years: form $0=$ no motor achievement; form 1 = head control; form 2 = sitting without help; form 3 =walking with support; form $4=$ walking independently. ? indicates patients not evaluated. The severity score for each family corresponds to the clinical score of most of the affected sibs. Mutations are numbered according to their position in the 7 exons of the PLP gene. Resulting amino acid changes are indicated in the single letter code, and their position in PLP is indicated (referring to transmembrane domains A to D, and intervening loop regions; see Figure 2). del, deletion; ins, insertion; asp, acceptor splice site; dsp, donor splice site; fs, frameshift.

*indicates mutations already reported.

\section{Correlation between disease severity and the type of amino-acid substitution resulting from missense mutations}

We assessed the correlation between the position of the aa substitution and disease severity. Changes in the PLP-specific B-C loop were responsible for the SPG phenotype. Ten of the 11 substitutions in the A-B extracytoplasmic loop and the 4 transmembrane segments of the PLP/DM20 protein caused severe forms of PMD. Five of the 11 substitutions in the $C-D$ loop caused severe PMD and 6 mild PMD. Three families (families 36, 37 and 49) had three different exon 4 mutations resulting in the substitution of the same amino acid, at position 202 in the PLP/DM20 protein (D202N, D202G, D202E). All the patients in these families had the most severe form of PMD (form 0). Two families (families 24, 53) had two different exon 5 mutations resulting in substitution of the same amino acid, at position 215 (P215S, P215A). All patients in these families had similar clinical severity (form 2). We 
A

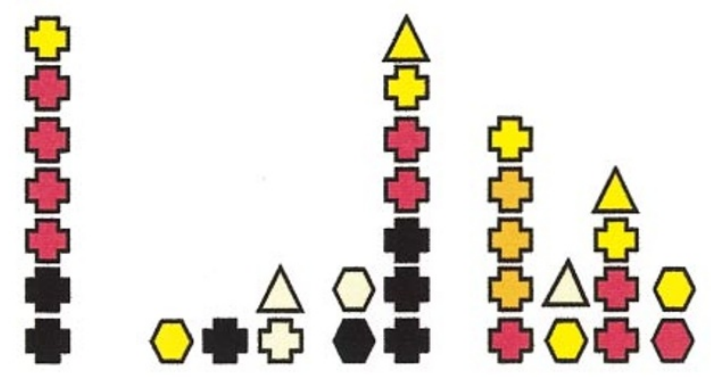

DNA

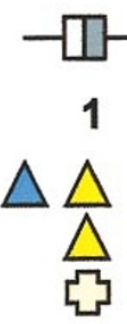

B

Exon
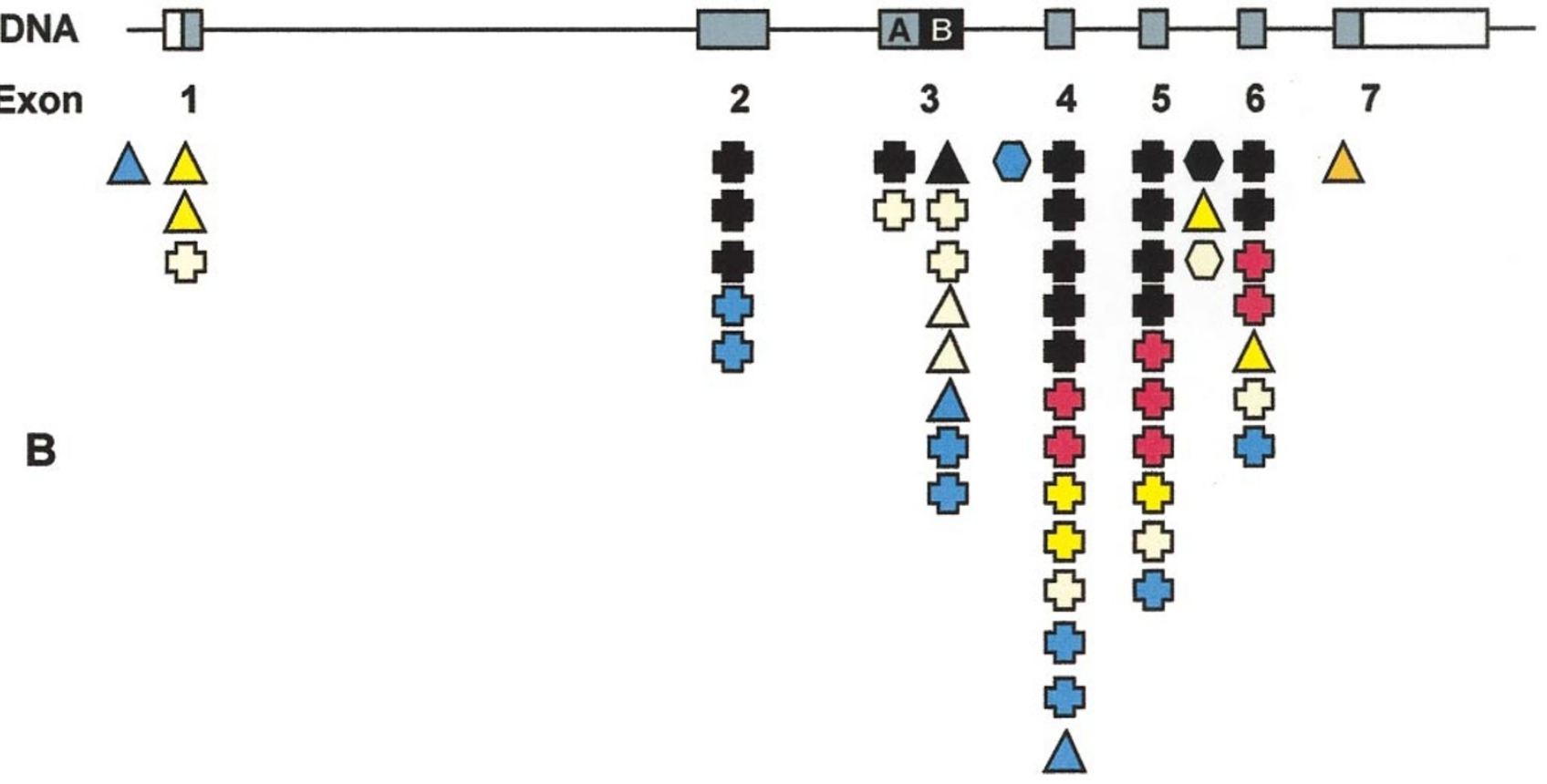

Figure 1 Severity of the disease according to the position and type of PLP mutation: A results obtained with the 33 families reported in this study; B results obtained with the 54 families reported in previous studies. Disease severity was scored according to the best motor function achieved between the ages of 1 and 10 years and is represented as follows: black, form $0=$ no motor achievement; red, form 1 = head control; orange, form 2 = sitting without help; dark yellow, form 3 = walking with support; light yellow, form 4 = walking independently. Undetermined forms (unreliable clinical data) are shown in blue. Type of PLP mutation found: missense, $\Delta$ nonsense, deletion/insertion and frameshift, splicing abnormalities.

analysed further the correlation between the degree of conservation of the substituted amino acid in DM proteins and the severity of PMD (Figure2). Replacement of a highly conserved amino acid, whatever the new amino acid, caused the most severe forms of PMD, whereas substitutions of less conserved amino acids caused milder forms. In two cases, a severe form of PMD was observed (Y174C and A247E), despite substitution of a poorly conserved amino acid. In both cases, the amino acid was in a transmembrane region (C and $\mathrm{D}$ respectively), close to a highly conserved amino acid and, in one case, the presence of a cysteine rather than the normal tyrosine may have severely affected amino acid interactions.

\section{Correlation between disease severity and splice site or stop mutations}

Four of the six mutations at splice sites were responsible for a very mild phenotype (type 4 or 3 ) with perfect intra-familial homongeneity (Table1). However, other mutations at the same splice site gave severe or mild forms of PLP-related diseases: family 26, with the most severe form of PMD and family 5, with the mildest SPG phenotype, had mutations in the same acceptor splice site of exon 4 and similarly, two PMD families, one with a severe form (1; family 62$)$ and the other with a mild form (3; family 221) had mutations in the same donor splice site of exon 6. One SPG2 patient had a 26-nucleotide deletion at the beginning of intron 5 . Three 


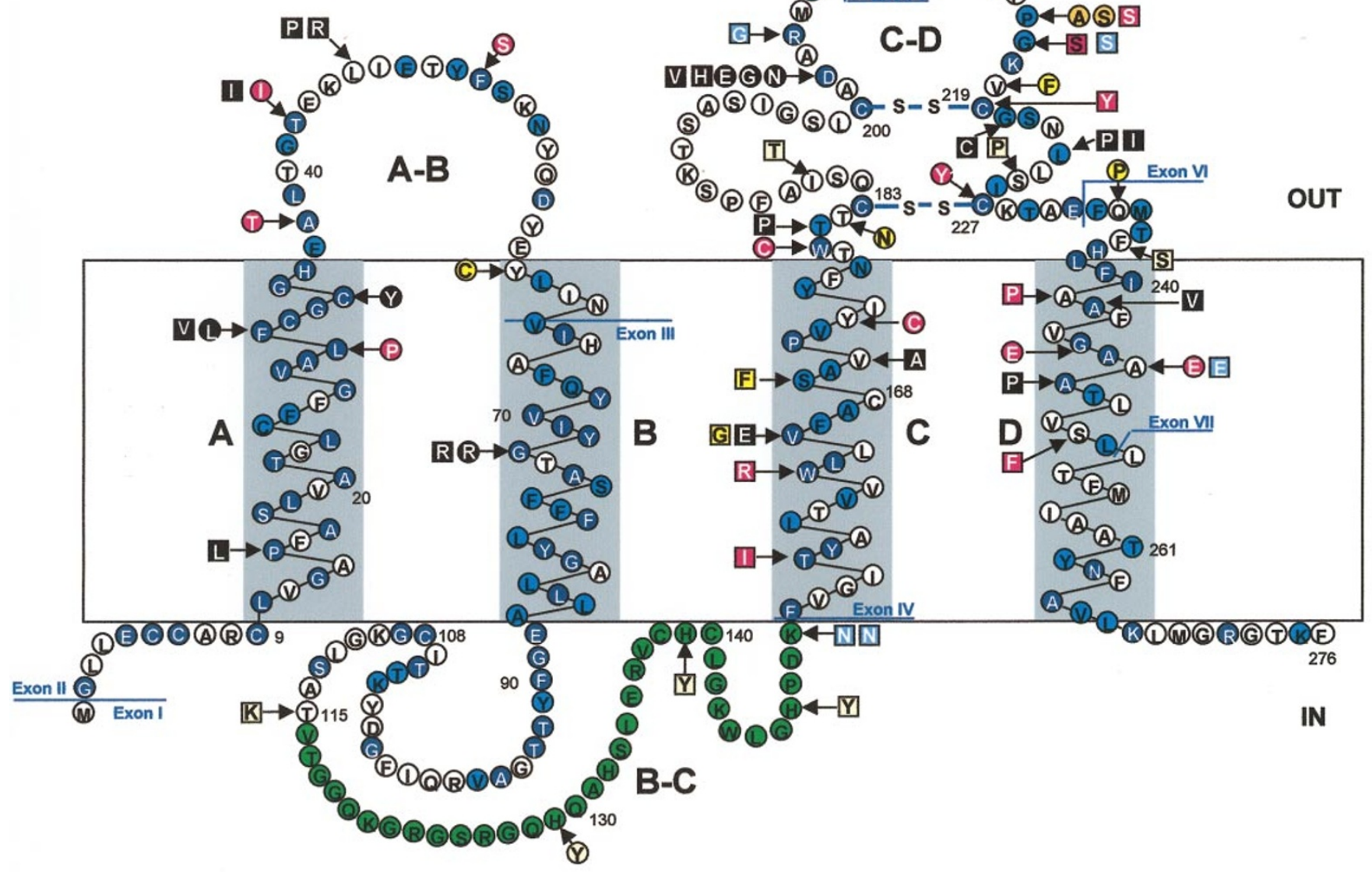

Figure 2 Disease severity according to the type of amino acid substitution in the PLP/DM 20 proteins. Schematic representation of the PLP protein with its four hydrophobic segments (A, B, C and D), two extracytoplasmic (A-B and C-D) and 1 intracytoplasmic (B-C) loop. ${ }^{3}$ The PLP protein has 276 amino acids. The 35 PLP-specific amino acids absent from the DM20 isoform are shown in green. The human DM20 amino acid sequence was compared with those of the DM20 of trout ${ }^{14}$ and chicken, ${ }^{15}$ the M6a, M6b proteins of humans ${ }^{16}$ and mice ${ }^{17}$ and the squalus $\mathrm{DM} \alpha, \mathrm{DM} \beta$ and $\mathrm{DM} \gamma$ proteins. ${ }^{18}$ Dark blue circles indicate highly conserved amino acids (90 to $100 \%$ identity), sky blue circles indicate partially conserved amino acids (60 to $89 \%$ identity) and white circles, non-conserved amino acids ( $<60 \%$ identity). The 23 missense mutations found are represented as circles. Missense mutations reported in previous studies are represented as squares. Disease severity was scored according to the same criteria and colour code described in Figure1.

deletion/insertion events (one in exon 3B, one in exon 4 and one in exon 6) were responsible for frameshifts with stop codons leading to the production of a truncated protein. One of these patients (SPG phenotype, family 9) had a PLP-specific mutation resulting in a normal DM20 and a truncated PLP lacking the $\mathrm{C}$-terminal region at the beginning of the $\mathrm{C}$ segment (stop after aa144). The other two patients had a mild form of PMD (families 3 and 182), with both PLP and DM20 proteins truncated after the D segment (at aa233 and 257, respectively). However, in one case, the frameshift changed 38 of the 58 aa of the C-D loop, and in the other it did not affect the C-D loop.

\section{Discussion}

The widespread use of cerebral MRI and CNS conduction velocity studies has transformed the recognition of human inherited defects of CNS myelination. However, the molecular defect has been identified only for PMD and SPG2, for which detection was easy due to their $\mathrm{X}$-linked inheritance. Both diseases involve the PLP gene, but they differ clinically in the onset and severity of motor disabilities. We investigated PMD and SPG families with no large PLP duplications or deletions by sequencing the coding regions and splice sites of PLP. We identified abnormalities in 54\% of PMD and $14 \%$ of SPG cases. Patients with PLP mutations presented a large 
range of clinical severity. There was a clinical continuum between the various forms, with the mildest forms of PMD similar to complicated forms of SPG. We used the best motor function achieved between the ages of 1 and 10 years to classify our patients. Disease severity was similar for members of the same family, which enabled us to score disease severity for each family. Disease severity was correl ated with the type of mutation. The severe forms of PMD were most frequently associated with missense mutations in exons 2 and 4 , leading to amino acid changes at positions highly conserved in other DM proteins. The mild forms of PMD were frequently caused by mutations resulting in the production of truncated proteins or by missense mutations. These missense mutations mostly affected exon 5, leading to the substitution of amino acids partly conserved in the extracytoplasmic C-D loop of PLP/DM 20 proteins. SPG was associated with splice site abnormalities or PLP-specific mutations.

We compared these results with those obtained by clinical analysis of the 52 families with duplications of the PLP gene. Most of the patients with duplications expressed form 2 $(42 \%)$ or $3(30 \%)$ of the disease. None had form 4 , and the most severe form 0 , was observed exclusively in patients with more than two copies of the PLP gene (un published data). In sharp contrast, in our family with a large deletion of the PLP gene leading to the absence of PLP/DM20 transcripts, one patient had form 3 and the other form 4 of the disease (unpublished data), consistent with another report. ${ }^{19}$ We pooled our results with those for the 54 PLP-mutated families (44PMD and 10SPG) with no large PLP duplications or deletions reported in previous studies (for references, see www.med.wayne.edu/neurology/plp.html), to give a total of 87 PLP mutations (73 PMD and 14SPG), 72\% of which were missense mutations. The total number of mutations for each exon was independent of the number of bases in that exon: excluding exon 1, the highest mutation/base ratios were obtained for exons5 (0.21), $6(0.16)$ and 4 (0.12) confirming that mutations most frequently affected the $C D$ region of the protein (63\%), particularly the C-D loop (39\%). In 42 (32 PMD and 10SPG) of the 54 families reported in previous studies, the clinical description was sufficient to classify patients according to our disease severity clinical score. We analysed the correlation between disease severity and the PLP mutation reported (Figures 1 and 2); the results were very similar to those obtained with our series of patients. In a total of 75 families with PLP mutations scored with our clinical criteria, 55\% were severe PMD, 27\% mild PMD and 18\% SPG. Mutations causing severe forms mapped in $56 \%$ of cases to exons 2 and 4 , in $36 \%$ to exons 5 and 6 , rarely to exon $3(8 \%)$, and never to exons 1 and 7. Mutations causing mild forms mapped in $57 \%$ of cases to exons 4 and 5 , in $31 \%$ to exons 1 and 6 and rarely to exons 2 and 7 (12\%). SPG mutations mapped in 54\% of cases to exon 3B, never to exons 2 and 7 and all other locations accounted for $9 \%$ each.

The quantitative fluorescent multiplex technique, ${ }^{8,20}$ makes possible the rapid and reliable detection of PLP duplication, the most frequent abnormality in patients with PMD (50\%). Testing for PLP point mutations is more time consuming but identified abnormal ities in 54\% of PMD cases with no PLP duplication. Our results and those obtained by analysis of previous studies suggest that clinical evaluation of disease severity can optimise point mutation searches by determining the order in which exons should be analysed: exons 2 and 4 , then 5 and 6 , and possibly $3 A$ for severe PMD; exons 5 and 4 , then 6 and 1 , and possibly 7 for mild PMD; exons 3 , and possibly 4, 5, 1, and 6 for SPG.

Seven missense or nonsense mutations affected the same amino acid of the PLP/DM 20 protein, as in our study. For the 6 missense mutations (F31, T42, G73, H202 and P215), disease severity was similar despite the fact that the aa change was different in three cases. In addition, the missense and nonsense mutations affecting Q233 resulted in the same mild form (3). The two different aa substitutions reported in the conservative aa223 (L223P and L223I), ${ }^{21}$ caused a severe form of PMD. However, severe forms of PMD were also reported in two mutations affecting the non-conservative aa 45 (L45P and L45R), ${ }^{21}$ suggesting that any change in the extracytoplasmic A-B loop is deleterious for oligodendrocytes. In contrast, changes in the very conservative valine165 caused either severe (V165E) or very mild (V165G) PMD. This might be due to differences in hydrophobicity changes for the affected transmembrane helix $C$ resulting from the substitution. ${ }^{21}$

We classified the phenotype of the PLP-specific nonsense mutation in exon 3B (W144X) $)^{22}$ as SPG, whereas the three patients were originally described as having a movement disorder (tremor, ataxia), apparently without spasticity, due to normal milestone achievement during the first year of life. In our series, SPG family 9 had a very similar PLP-specific abnormality: the five affected patients expressed a classical PMD3 or SPG phenotype. For five previously reported SPG families, mutations were outside the PLP-specific exon 3B. One was a nonsense mutation in the ATG of the first exon, resulting in the absence of PLP and DM20. ${ }^{23} \mathrm{~A}$ mild PMD3 phenotype has been also reported for the other two nonsense mutations described in the first coding bases ATGG (delG3, delG4). ${ }^{24,25}$ The mutation at the end of exon 3A (ACA-AAA) has been classified as a form of SPG. ${ }^{26}$ This PLP mutation outside exon 3B may be responsible for a T115K amino acid substitution in PLP/DM20 but may also disturb the splicing of exon 3 resulting in the absence of DM20. The three other non PLP-specific mutations responsible for SPG affected nonconservative aa of the C-D loop, for which we found three mutations with a PMD 3 phenotype. Thus, the absence of PLP/DM 20, PLP-specific mutations and substitutions of non conserved amino acids in the C-D loop are responsible for the mildest form of PMD, which is clinically similar to complicated forms of SPG.

Mutations resulting in the production of truncated proteins were most frequently associated with the mildest forms of dysmyelinating disease (76\% of PMD3 and SPG cases). 
However, the mutation in the acceptor splice site of exon 5 in the jimpy mouse PLP mutant, results in a severe phenotype in which myelin is almost totally absent and the oligodendrocytes die. ${ }^{6}$ In humans, point mutations at a single splice site were associated with different clinical phenotypes. Differences in phenotype could be due to different DM20 mRNA defects (Cailloux, personal communication). PLP mutant mice provide an accurate model of human diseases, with similar variability in the severity of phenotypes. ${ }^{9}$ This model suggests that the severe impairment in the achievement of normal milestones during the first year of life that distinguishes PMD from SPG may result from a deficit in the number of mature oligodendrocytes, the severity of PMD being correlated with the number of surviving oligodendrocytes. ${ }^{27-30}$ The cellular events responsible for differences in the survival of myelinating oligodendrocytes are unknown. The mechanisms by which abnormal PLP products may be deleterious to the cell include the loss of normal function, a gain of function in which the altered protein becomes cytotoxic, and perturbation of intracellular trafficking in oligodendrocytes. In vitro experiments ${ }^{31}$ have suggested that 'severe PMD' phenotypes are associated with an absence of PLP and DM20 at the cell surface whereas 'milder' phenotypes are associated with normal level s of DM20 at the membrane and accumulation of PLP in the endoplasmic reticulum. However, the clinical criteria used to evaluate the severity of the phenotype were not defined. Studies with mutant mice have suggested that clinical severity depends less on capacity of PLP/DM 20 to reach the membrane than on their capacity to interact with other proteins during transit, with the accumulation of misfolded proteins increasing oligodendrocyte death by apoptosis. ${ }^{32}$

In PLP-related defects of myelination, PMD involves impaired motor development during the first year of life, related to the death of mature oligodendrocytes. In contrast, SPG involves almost normal motor milestone achievement followed by progressive gait abnormalities resulting from abnormal myelin compaction, without the death of oligodendrocytes. The clinical continuum from the most severe forms of PMD to the mildest SPG phenotype (Figure3) results from the great variability in the number of surviving oligodendrocytes. However, disease severity is similar for patients with the same mutation. Disease severity appears to be correlated with the amount of PLP/DM20 produced: absence of these proteins leads to the mildest phenotype, whereas overproduction results in mild or severe forms of PMD depending on the level of PLP transcription. Finally, severity is correlated with the type of protein mutated: mutated PLP with normal DM20 causes the mildest phenotype, whereas mutated DM20 causes mild or severe forms

\section{Clinical severity}

\begin{tabular}{|c|c|c|c|}
\hline \multicolumn{2}{|l|}{ SEVERE PMD } & MILD PMD & \multirow{2}{*}{$\begin{array}{l}\text { SPG } \\
\text { FORM } 4\end{array}$} \\
\hline FORM 0 & & FORM 3 & \\
\hline \multirow[t]{2}{*}{$\begin{array}{l}\text { point mutations in } \\
\text { highly conserved region } \\
\text { of PLP }\end{array}$} & & \multirow[t]{2}{*}{$\begin{array}{l}\text { point mutations in } \\
\text { partially conserved } \\
\text { region of PLP }\end{array}$} & $\begin{array}{l}\text { point mutations in } \\
\text { non conserved } \\
\text { region of PLP }\end{array}$ \\
\hline & & & $\begin{array}{l}\text { PLP-specific } \\
\text { mutations }\end{array}$ \\
\hline Overexpression & of & PLP/DM20 & Absence of PLP/DM20 \\
\hline
\end{tabular}

\section{Oligodendrocyte death}

Figure 3 Genotype-phenotype correlation in PLP-related diseases. The large spectrum in disease severity, with a continuum from the most severe forms of PMD to the mildest SPG phenotype, is related to the type of PLP mutation and the number of surviving oligodendrocytes. PMD, Pelizaeus-Merzbacher Disease; SPG, X-linked spastic paraplegia type2. 
depending on changes in the interactions between mutated proteolipid protein monomers. Substitutions of very conserved aa and truncated proteins with large changes in C-terminal structure have the largest effect on the intracellular interactions of mutated proteins. However, the cellular mechanisms responsible for oligodendrocyte death are unknown.

\section{Acknowledgements}

This work was supported by grants from the Institut National de la Santé et de la Recherche Médicale (Réseau Recherche Clinique, PROGRES), European Leukodystrophies Association (ELA France), the EU Biomed 2 (BMH4-961405) project and the Jean Pierre and Nancy Boespflug Foundation for Myopathic Research.

\section{References}

1 Boulloche J, Aicardi J: Pelizaeus-Merzbacher disease: clinical and nosological study. J Child Neurol 1986; 1: 233-239.

2 Boespflug-Tanguy O, Mimault C, Melki J et al: Genetic homogeneity of Pelizaeus-M erzbacher disease: tight linkage to the proteolipoprotein locus in 16 affected families. PMD Clinical Group. Am Hum Genet 1994; 55: 461-467.

3 Weimbs T, Stoffel W: Proteolipid protein (PLP) of CNS myelin: positions of free, disulfide-bonded, and fatty acid thioester-linked cysteine residues and implications for the membrane topology of PLP. Biochemistry 1992; 31: 12289-12296.

4 LeVine SM, Wong D, Macklin WB: Developmental expression of proteolipid protein and DM20 mRNAs and proteins in the rat brain. Dev Neurosci 1990; 12: 235-250.

5 Yang X, Skoff RP: Proteolipid protein regulates the survival and differentiation of oligodendrocytes. J Neurosci 1997; 17: 2056-2070.

6 Nave KA: Neurological mouse mutants and the genes of myelin. J Neurosci Res 1994; 6: 607-612.

7 Sistermans EA, DeCoo RF, DeWijsIJ, Van Oost BA: Duplication of the proteolipid protein gene is the major cause of PelizaeusMerzbacher disease. Neurology 1998; 50: 1749-1754.

8 Mimault C, Giraud G, Courtois V et al: Proteolipoprotein gene analysis in 82 patients with sporadic Pelizaeus-Merzbacher Disease: duplications, the major cause of the disease, originate more frequently in male germ cells, but point mutations do not. The Clinical European Network on Brain Dysmyelinating Disease. Am J Hum Genet 1999; 65: 360-369.

9 Nave KA, Boespflug-Tanguy O: X-linked developmental defects of myelination: From mouse mutants to human genetic diseases. Neuroscientist 1996; 2: 33-43.

10 Saugier-Veber $P$, Munnich A, Bonneau $D$ et al: X-linked spastic paraplegia and Pelizaeus-Merzbacher disease are allelic disorders at the proteolipid protein locus. Nat Genet 1994; 6: 257-261.

11 Kobayashi $\mathrm{H}$, Hoffman $\mathrm{E}, \mathrm{Marks} \mathrm{H}$ : The rumpshaker mutation in spastic paraplegia. Nat Genet 1994; 7: 351-352.

12 Osaka H, Kawanishi C, Inoue K et al: Novel nonsense proteolipid protein gene mutation as a cause of $X$-linked spastic paraplegia in twin males. Biochem Biophys Res Commun 1995; 215: 835-841.

13 Pham-Dinh D, Popot JL, Boespflug-Tanguy $O$ et al: PelizaeusMerzbacher disease: a valine to phenylalanine point mutation in a putative extracellular loop of myelin proteolipid. Proc Natl Acad Sci USA 1991; 88: 7562-7566.

14 Tang S, Panno JP, McKeown BA: Cloning and expression of the proteolipid protein DM20 CDNA from the brain of the rainbow trout, Oncorhynchus mykiss. Brain Res Mol Brain Res 1996; 41 : 134-139.
15 Schliess F, Stoffel W: Evolution of the myelin integral membrane proteins of the central nervous system. Biol Chem Hoppe Seyler 1991; 372: 865-874.

16 Olinsky S, Loop BT, DeKosky A et al: Chromosomal mapping of the human M6 genes. Genomics 1996; 33: 532-536.

17 Yan Y, Lagenaur C, Narayanan V: Molecular cloning of M6: identification of a PLP/DM20 gene family. Neuron 1993; 3: 423-431.

18 Kitagawa K, Sinoway MP, Yang C, Gould RM, Coldman DR: A proteolipid protein genefamily: expression in sharks and rays and possible evolution from an ancestral gene encoding pore-forming polypeptides. Neuron 1993; 11: 433-448.

19 Raskind WH, Williams CA, Hudson LD, Bird TD: Complete deletion of the proteolipid protein gene (PLP) in a family with X-linked Pelizaeus-M erzbacher disease. Am J Hum Genet 1991; 49: $1355-1360$

20 Woodward K, Kendall E, Vetrie D, Malcolm S: Pelizaeus-Merzbacher disease: identification of Xq22 proteolipid-protein duplications and characterization of breakpoints by interphase FISH. Am J Hum Genet 1998; 63: 207-217.

21 Hodes ME, Zimmerman AW, Aydanian A et al: Different mutations in the same codon of the proteolipid protein gene, PLP, may help in correlating genotype with phenotype in Pelizaeus-Merzbacher disease/X-linked spastic paraplegia (PMD/SPG2). Am J Med Genet 1999; 15: 132-139.

22 Hodes ME, Blank CA, Pratt VM, Morales J, Napier J, Dlouhy SR: Nonsense mutation in exon 3 of the proteolipid protein gene (PLP) in a family with an unusual form of Pelizaeus-Merzbacher disease. Am J Med Genet 1997; 69: 121-125.

23 Sistermans EA, De Wijs IJ, De Coo RF, Smit LM, Menko FH, Van Oost BA: A (G-to-A) mutation in the initiation codon of the proteolipid protein gene causing a relatively mild form of Pelizaeus-M erzbacher disease in a Dutch family. Hum Genet 1996; 97: 337-339.

24 Garbern JY, Cambi F, Tang XM et al: Proteolipid protein is necessary in peripheral as well as central myelin. Neuron 1997; 19: 205-218.

25 Hodes ME, Pratt VM, Dlouhy SR: Genetics of Pelizaeus-Merzbacher disease. Dev Neurosci 1993; 15: 383-394.

26 Nance MA, Boyadjiev S, Pratt VM, Taylor S, Hodes ME, Dlouhy SR: Adult-onset neurodegenerative disorder due to proteolipid protein gene mutation in the mother of a man with PelizaeusMerzbacher disease. Neurology 1996; 47: 1333-1335.

27 Vermeesch MK, Knapp PE, Skoff RP, Studzinski DM, Benjamins JA: Death of individual oligodendrocytes in jimpy brain precedes expression of proteolipid protein. Dev Neurosci 1990; 12: 303-315.

28 Gow A, Southwood CM, Lazzarini RA: Disrupted proteolipid protein trafficking results in oligodendrocyte apoptosis in an animal model of Pelizaeus-Merzbacher disease. I Cell Biol 1998; 140: 925-934.

29 Schneider A, Montague $P$, Griffiths I et al: Uncoupling of hypomyelination and glial cell death by a mutation in the proteolipid protein gene. Nature 1992; 358: 758-761.

30 Klugmann $\mathrm{M}$, Schwab MH, Puhlhofer A et al: Assembly of CNS myelin in the absence of proteolipid protein. Neuron 1997; 18: 59-70.

31 Gow A, Lazzarini RA: A cellular mechanism governing the severity of Pelizaeus-M erzbacher disease. Nat Genet 1996; 13: 422-428.

32 Jung $M$, Sommer I, Schachner M, Nave KA: Monoclonal antibody O10 defines a conformationally sensitive cell-surface epitope of proteolipid protein (PLP): evidence that PLP misfolding underlies dysmyelination in mutant mice. J Neurosci 1996; 16: 7920-7929. 